\title{
Capacity Analysis and Optimization in Heterogeneous Network with Adaptive Cell Range Control
}

\author{
Xinyu Gu, ${ }^{1}$ Xin Deng, ${ }^{1}$ Qi Li, ${ }^{1}$ Lin Zhang, ${ }^{1}$ and Wenyu $\mathrm{Li}^{2}$ \\ ${ }^{1}$ Beijing University of Posts and Telecommunications, Beijing 100876, China \\ ${ }^{2}$ Beijing Key Lab of New Generation Broadband Wireless Mobile Communication Technology, Standard and Verification, \\ China Academy of Telecommunication Research, Beijing 100191, China \\ Correspondence should be addressed to Xinyu Gu; guxinyu.bj@gmail.com
}

Received 20 February 2014; Revised 7 April 2014; Accepted 8 April 2014; Published 29 April 2014

Academic Editor: Xiang Zhang

Copyright (C) 2014 Xinyu Gu et al. This is an open access article distributed under the Creative Commons Attribution License, which permits unrestricted use, distribution, and reproduction in any medium, provided the original work is properly cited.

\begin{abstract}
As an attractive means of expanding mobile network capacity, heterogeneous network is regarded as an important direction of mobile network evolution. To increase the capacity of, for example, hot spots, a typical scenario in heterogeneous network is that the coverage areas of low power nodes (LPNs) are overlapped with macrocell. To increase the utilization of small cells generated by LPNs, cell range extension (CRE) is used to extend the coverage of the small cells by adding cell specific offset (CSO) to small cells during cell selection procedure. The value of CSO, however, needs to be set carefully. In this paper, the capacity of users in macrocells, users in small cells, and users in range extension areas is analyzed thoroughly in conditions with and without CRE. Based on the analysis, an adaptive CSO updating algorithm is proposed. The proposed algorithm updates the CSO value periodically by predicting the overall capacity and a new CSO value is selected which can give the optimal overall capacity. The proposed algorithm is evaluated by system-level simulations. Simulation results indicate that the proposed algorithm can ensure a nearly optimal performance in all tested traffic load situations.
\end{abstract}

\section{Introduction}

A heterogeneous network is typically composed of multiple radio access technologies, transmission solutions, and base stations of varying transmission power [1]. To meet the increasing traffic demands and fulfilling users' high expectations for mobile broadband, heterogeneous network has been included in the evolution version of long-term evolution (LTE) radio access technology, named LTE-advanced, in the third generation partnership project (3GPP) $[2,3]$ together with higher order multiple input and multiple output (MIMO) [4], carrier aggregation (CA) [5], and coordinated multipoint transmission and reception (CoMP) [6].

In a heterogeneous network, one typical scenario is that the coverage area of the low power nodes (LPN) and macrocells are overlapped with each other. Considering the case that the macrocell's carrier frequencies are reused throughout the network, the interference between macrocells and LPNs is a very essential issue which needs to be considered in order to optimize the network performance and end-user experience especially with cell range extension (CRE) of LPNs. Poorly planned LPNs location or configuration not only results in unsatisfactory experience in its coverage area but also may cause performance degradation and overload state in macrocell [7]. CRE was proposed in 3GPP in Release $10[8]$ with the purpose to enlarge the coverage area of LPNs and increase the served users in LPNs so as to explore the system resource (e.g., power, spectrum) more efficiently.

CRE is obtained by setting a cell selection offset (CSO) to LPNs during cell selection/reselection procedure so that the end-user would select the LPN with the higher priority. However, when extending the served area of LPNs, the users that locate in the extended range could suffer from severe interference from the macro base station due to the difference between the transmit power of the macro base stations and the LPNs. This is illustrated by Figure 1.

As discussed above, there is a trade-off between the LPN offloading ratio and the user experienced interference in 


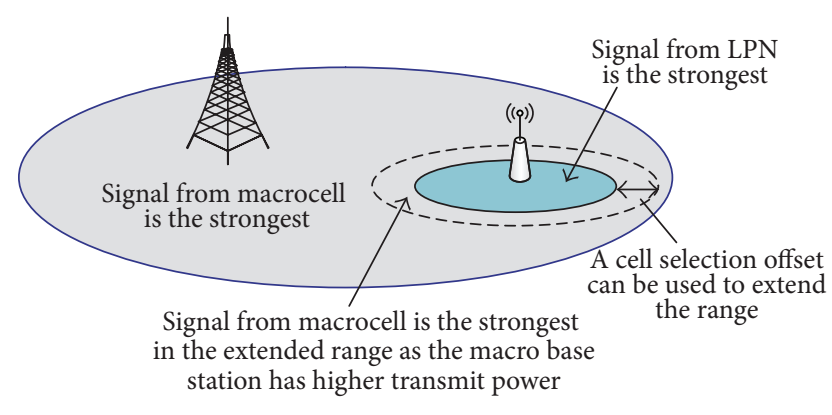

Figure 1: Illustration of cell range extension.

LPNs especially in the extended LPN range. Therefore, how to properly set CSO is very necessary to be studied. In previous investigations, CSO was usually decided through simulations, for example, in [9], and some fixed value was proposed in 3 GPP based on system-level simulations. However, simulations cannot cover the varying situations in reality. Therefore, it is necessary to discuss the optimal CSO setting from theoretical point of view. In [10], a semianalytic tool for investigating the capacity and fairness of heterogeneous networks with range extension was provided. With this tool, the sum capacity and other metrics of interest can be evaluated as a continuous function of the CRE. It is an important attempt to optimize CSO setting through an analytical way than in pure simulation. However, there are still some limitations in [10]. Firstly, in the analysis the relationship between the cell load and the signal to interference and noise ratio (SINR) was not considered. Secondly, the load estimation of each cell was simply through the number of users in each cell, but the number of users may not be the most suitable metric to directly represent the actual cell load. For example, the traffic volume of each user also plays an important role in actual cell load. In [11], spectral efficiency was analyzed for heterogeneous network with CRE, and the main conclusion was that the CRE biasing deteriorates the outage and rate of the overall network by lowering the SINR if assuming full queues at all base stations. However, this assumption may not be true in reality and needs to be relaxed as pointed out by [11]. Besides theoretical analysis, considering the varying conditions in the real wireless network, self-organizing network (SON) is a powerful tool to adaptively adjust the network configuration parameters to realize self-optimizing [12-14]. In scenario of heterogeneous network, SON is still an effective way to achieve network self-configuration and self-optimizing [15]. There were some attempts to use SON to adaptively adjust the CSO setting, for example, in $[16,17]$. In [16] an adaptive algorithm to decide the CSO value was proposed based on the end-user performance feedback. However, there are limitations with the algorithm in [16]. Firstly, the number of users was used to decide the load in each cell which is not accurate enough in reality as pointed out. Secondly, it was proposed to use cell edge user throughput and average user throughput as inputs to the adaptation algorithm. However, both average user throughput and cell edge user throughput cannot be obtained from the network side directly. It requires feedback from users and increases uplink signaling overhead. In [17], the authors proposed an adaptive CRE controlling technique that improves the cell edge user throughput in heterogeneous network, in which UE can automatically choose an optimal $\mathrm{CSO}$ from either $\mathrm{CSO}_{\text {high }}$ or $\mathrm{CSO}_{\text {low }}$. However, the setting of $\mathrm{CSO}_{\text {high }}$ and $\mathrm{CSO}_{\text {low }}$ was not sufficiently discussed.

In this paper, the capacity of users in macrocells, users in small cells, and especially users in range extension areas is analyzed thoroughly in conditions with and without CRE. In the analysis, cell load is estimated based on the resource block (RB) utilization ratio. Furthermore, the relationship between cell load and SINR is considered in the analysis. Conditions to achieve higher capacity by CRE are derived, respectively, for users served by macrocell and LPNs. Based on the analysis, an adaptive CSO updating algorithm is proposed. The proposed algorithm updates the CSO value periodically by predicting the overall capacity and a new CSO value is selected which can give the optimal overall capacity.

\section{Capacity Analysis with Cell Range Extension}

In this section, the capacities of users in macrocells and in small cells are analyzed especially for users in CRE region. Users in CRE region refer to users whose serving cell changes with CRE, for example, served by macrocell without CRE but served by LPN with CRE.

In this paper, a mathematical framework for quantitative investigation of self-optimizing wireless network (SON) [18, $19]$ is partly reused for the basis of the analysis.

(i) A network layout by network nodes, either macrocells or LPNs, defining a cell $c$ at the position $\vec{q}_{c}$;

(ii) user equipment (UE) $u$ located at position $\vec{q}_{u}$ : in dynamic simulation the user positions can change over simulation time;

(iii) UE $u$ is served by cell $c=S(u)$, where $S(u)$ is the connection function, and every user is connected exactly to a single base station (BS);

(iv) a pathloss mapping, $L_{c}\left(\vec{q}_{u}\right)$, defined by the positions of user $u$ relative to a cell $c$ : the pathloss mapping takes all position-dependent channel model effects into account, for example, distance-dependent pathloss, shadowing, and angle-dependent antenna patterns;

(v) a cell load $\rho_{c}$, which defines the ratio of used resources in LTE physical resource blocks (PRBs) to all available resources in a given cell;

(vi) a transmit power $P_{c}$ for cell $c$ and thermal noise $N$.

Based on the above definitions, the SINR of user $u$, denoted by $\gamma_{u}$, with serving cell $S(u)$ can be written as

$$
\gamma_{u}=\frac{P_{S(u)} L_{S(u)}\left(\vec{q}_{u}\right)}{N+\sum_{c \neq S(u)} \rho_{c} P_{c} L_{c}\left(\vec{q}_{u}\right)} .
$$

2.1. Mechanism for Cell Range Extension. Typically, the served area of a LPN is smaller than the served area of a macro base station. This is in line with the criterion of cell selection. For 
cell selection, usually the UE measures the reference signal received power (RSRP) from each cell and selects the serving cell with the maximum received signal power as shown by

$$
S(u)=\arg \max _{\{c\}}\left(\operatorname{RSRP}_{u, c}\right)=\arg \max _{\{c\}}\left(P_{c} \cdot L_{c}\left(\vec{q}_{u}\right)\right) .
$$

In (2), UE $u$ selects cell $c$ as its serving cell $S(u)$ because the RSRP from cell $c$ is the maximum among the measurements from different cells.

In homogeneous network, the cell with the maximum RSRP is usually the cell with the best path-gain to this UE; however, in heterogeneous network where the deployed base stations have different transmit powers, the cell with maximum RSRP may no longer be the cell with the best path-gain to the UE. As shown in (2), the RSRP of user $u$ received from cell $c$ is the transmit power of cell $c$ multiplied by path-gain $\left(L_{c}\right)$ between cell $c$ and user $u$ (at the position $\left.\vec{q}_{u}\right)$. Considering a typical transmit power of macrocell and picocell as $43 \mathrm{dBm}$ and $30 \mathrm{dBm}$, respectively, only UE whose path-gain to the picocell is $13 \mathrm{~dB}$ better than the path-gain to macrocell can select the picocell as its serving cell. This leads to a small number of served users in LPNs.

To cope with this issue, CRE is proposed for heterogeneous network in 3 GPP. With CRE, a cell specific bias, CSO, is added to the measured RSRP during cell selection procedure as shown in

$$
S(u)=\arg \max _{\{c\}}\left(\operatorname{RSRP}_{u, c}+\mathrm{CSO}^{c}\right) .
$$

The CSO value is usually zero for macrocell and a positive value for a LPN.

2.2. Capacity Analysis with Cell Range Extension. Assuming ideal link adaptation, which means that the most suitable modulation and coding scheme (MCS) is selected according to the SINR of user $u$, the achievable data rate can be represented by the Shannon capacity as

$$
R\left(\gamma_{u}\right)=\log _{2}\left(1+\gamma_{u}\right)
$$

Here $R\left(\gamma_{u}\right)$ is the achievable data rate if user $u$ is scheduled. Considering the normal case that more than one user is connecting to the same cell and sharing the resource in the same cell and by assuming a round robin scheduling strategy, the achievable capacity can be represented by

$$
C(u)=\left(1-\rho_{S(u)}\right) \log _{2}\left(1+\gamma_{u}\right),
$$

where $\rho_{S(u)}$ is the load in the serving cell $S(u)$. Intuitively, users in a cell with high load (large $\rho_{c}$ ) have lower probability to get chance to be scheduled, and even if the SINR of this user is good, the final capacity is still not high.

2.2.1. Capacity Analysis for Users in CRE Region. In this section, capacity of users in the CRE region is analyzed. For these users, before a CSO value is applied, the serving cell of these users is the macrocell, and after the CSO value is applied, the serving cell of these users is the LPN. We analyze the capacity change of these users with and without CRE.
Denote the capacity of user $u$ in CRE region by $C_{\mathrm{CRE}}(u)$. For these users, the serving cell $S(u)$ will change with and without CRE. Represent the serving cell before applying CSO as $S^{(m)}(u)$ and the serving cell after applying CSO as $S^{(p)}(u)$. The SINR of user $u$ before applying CSO is $\gamma_{u, \text { CRE}}^{(m)}$,

$$
\gamma_{u, \mathrm{CRE}}^{(m)}=\frac{P_{S^{(m)}(u)} L_{S^{(m)}(u)}\left(\vec{q}_{u}\right)}{N+\sum_{c \neq S^{(m)}(u)} \rho_{c} P_{c} L_{c}\left(\vec{q}_{u}\right)} .
$$

To facilitate further analysis, the interference from the picocell, which is the serving cell after applying CSO, is separated from the overall interference. Therefore, the SINR of user $u$ before applying CSO is

$$
\begin{aligned}
\gamma_{u, \mathrm{CRE}}^{(m)}= & \frac{P_{S^{(m)}(u)} L_{S^{(m)}(u)}\left(\vec{q}_{u}\right)}{N+\sum_{c \neq S^{(m)}(u)} \rho_{c} P_{c} L_{c}\left(\vec{q}_{u}\right)} \\
= & \left(P_{S^{(m)}(u)} L_{S^{(m)}(u)}\left(\vec{q}_{u}\right)\right) \\
& \times\left(\begin{array}{c}
N+\sum_{c \neq S^{(m)}(u)} \rho_{c} P_{c} L_{c}\left(\vec{q}_{u}\right) \\
c \neq S^{(p)}(u) \\
+\rho_{S^{(p)}(u)} P_{S^{(p)}(u)} L_{S^{(p)}(u)}\left(\vec{q}_{u}\right)
\end{array}\right)^{-1} .
\end{aligned}
$$

Suppose the SINR of user $u$ after applying CSO is $\gamma_{u, \mathrm{CRE}}^{(p)}$;

$$
\gamma_{u, \mathrm{CRE}}^{(p)}=\frac{P_{X^{(p)}(u)} L_{X^{(p)}(u)}\left(\vec{q}_{u}\right)}{N+\sum_{c \neq X^{(p)}(u)} \rho_{c} P_{c} L_{c}\left(\vec{q}_{u}\right)} .
$$

With CRE, the received power from the previous serving cell becomes the interference. Similarly, the interference from the macrocell, which is the serving cell before applying CSO, is extracted from the overall interference for analysis. The SINR of user $u$ after applying CSO could be written as

$$
\begin{aligned}
\gamma_{u, \mathrm{CRE}}^{(p)}= & \frac{P_{S^{(p)}(u)} L_{S(u)}\left(\vec{q}_{u}\right)}{N+\sum_{c \neq S(u)} \rho_{c} P_{c} L_{c}\left(\vec{q}_{u}\right)} \\
= & \left(P_{S^{(p)}(u)} L_{S^{(p)}(u)}\left(\vec{q}_{u}\right)\right) \\
& \times\left(\begin{array}{c}
N+\sum_{\substack{c \neq S^{(m)}(u) \\
c \neq S^{(p)}(u)}} \rho_{c} P_{c} L_{c}\left(\vec{q}_{u}\right) \\
+\rho_{S^{(m)}(u)} P_{S^{(m)}(u)} L_{S^{(m)}(u)}\left(\vec{q}_{u}\right)
\end{array}\right) .
\end{aligned}
$$


TABLE 1: System evaluation assumptions and parameters.

\begin{tabular}{ll}
\hline Parameters & Values/descriptions \\
\hline $\begin{array}{l}\text { Macro base station } \\
\text { deployment }\end{array}$ & $\begin{array}{l}\text { Site to site distance: } 500 \mathrm{~m} \\
\text { Height: } 40 \mathrm{~m} \text { above ground } \\
\text { Tx power: } 46 \mathrm{dBm}\end{array}$ \\
\hline & 6 LPNs per macrocell on average \\
& $\begin{array}{l}\text { Height: } 5 \mathrm{~m} \text { above ground } \\
\text { Tx power: } 30 \mathrm{dBm}\end{array}$ \\
LPN deployment & Cell radius: $30 \mathrm{~m}$ \\
\hline Propagation model & ITU UMa [20] \\
\hline Antenna model & Macro: 3GPP [21] \\
\hline Transmission scheme & LPN: dipole \\
\hline Scheduler & SU-MIMO 2Tx 2Rx \\
\hline Traffic type & Proportional fair \\
\hline $\begin{array}{l}\text { Number of users per } \\
\text { macrocell }\end{array}$ & Download \\
\hline User distribution & 75 \\
\hline
\end{tabular}

For a user in the cell range extension area, although the selected serving cell is the picocell, the received power from macro base station is still larger than received signal power from picocell. This means that, for these users, it is true that

$$
P_{S^{(m)}(u)} L_{S^{(m)}(u)}\left(\vec{q}_{u}\right) \geq P_{S^{(p)}(u)} L_{S^{(p)}(u)}\left(\vec{q}_{u}\right) .
$$

Considering the most common case that the load in the macrocell is not lower than that in the picocell, comparing (7) and (9), it can be concluded that the UE who are "forced" into picocells by range extension will suffer from lower SINR. That is

$$
\gamma_{u, \mathrm{CRE}}^{(p)} \leq \gamma_{u, \mathrm{CRE}}^{(m)}
$$

For example, Figure 2 clearly shows the SINR loss of users in the cell range extension area. In Figure 2, the cell selection offset is $6 \mathrm{~dB}$ and more simulation settings can be found in Table 1.

Based on the analysis of the change of SINR of users in cell range extension region, the capacity of these users with and without cell range extension is further analyzed. Suppose the capacity of user $u$ before and after applying CSO is $C_{\mathrm{CRE}}^{(m)}(u)$ and $C_{\mathrm{CRE}}^{(p)}(u)$, respectively:

$$
\begin{aligned}
& C_{\mathrm{CRE}}^{(m)}(u)=\left(1-\rho_{S^{(m)}(u)}\right) \log _{2}\left(1+\gamma_{u, \mathrm{CRE}}^{(m)}\right), \\
& C_{\mathrm{CRE}}^{(p)}(u)=\left(1-\rho_{S^{(p)}(u)}\right) \log _{2}\left(1+\gamma_{u, \mathrm{CRE}}^{(p)}\right) .
\end{aligned}
$$

Comparing (12a) and (12b), it can be seen that although the SINR is expected to be reduced $\left(\gamma_{u, \text { CRE }}^{(p)} \leq \gamma_{u \text {, CRE }}^{(m)}\right)$ for users in CRE region after range extension, the capacity of these users depends on the cell load of their serving cells as well. If the cell load in the picocell is below the cell load in

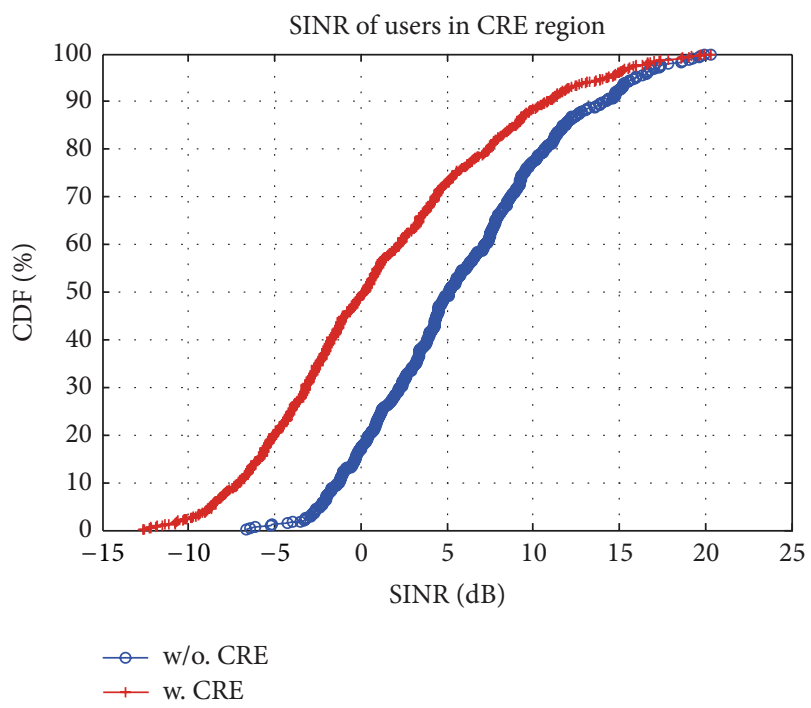

FIGURE 2: SINR of users in range extension area.

the macrocell by certain extent, although there is an expected SINR loss, the capacity of users in the CRE region can still expect an increase:

$$
\begin{gathered}
C_{\mathrm{CRE}}^{(p)}(u)>C_{\mathrm{CRE}}^{(m)}(u), \\
\left(1-\rho_{S^{(p)}(u)}\right) \log _{2}\left(1+\gamma_{u, \mathrm{CRE}}^{(p)}\right)>\left(1-\rho_{S^{(m)}(u)}\right) \log _{2}\left(1+\gamma_{u, \mathrm{CRE}}^{(m)}\right) .
\end{gathered}
$$

Therefore, we can derive the theorem below to show the condition that ensures a gain in capacity of users in CRE region.

Theorem 1. The capacity of users in region of CRE can be increased if and only if the cell load in the original serving macrocell before CRE satisfies the inequality as below:

$$
\rho_{S^{(m)}(u)}>1-\left(1-\rho_{S^{(p)}(u)}\right) \frac{\log _{2}\left(1+\gamma_{u, C R E}^{(p)}\right)}{\log _{2}\left(1+\gamma_{u, C R E}^{(m)}\right)} .
$$

Comparing the role of SINR and cell load in capacity, it can be seen that the cell load linearly contributes to the capacity while SINR contributes in logarithmic scale. Therefore, the contribution from cell load plays an even more important role in capacity than SINR does and needs to be treated seriously.

To summarize, on one hand, for users in the CRE region, usually the SINR decreases due to the suboptimal received signal power and high interference from macrocell; on the other hand, CRE may also bring benefit in terms of available resource (e.g., frequency, time, and power) to these users. More resource to a user means, for example, more opportunity to be scheduled by the network, which eventually contributes to the user capacity. 
Suppose the total capacity of users in CRE region is $C_{\mathrm{CRE}}$, and the number of users in the CRE region is $N_{R}$; we have

$$
C_{\mathrm{CRE}}=\sum_{u=1}^{N_{R}} C_{\mathrm{CRE}}(u) \text {. }
$$

2.2.2. Capacity Analysis for Users in Macrocell. In this section, the capacity of users who are served by the macrocell consistently before and after range extension is analyzed. For these users, although the serving cell is unchanged, the SINR and capacity are impacted anyway by range extension.

Suppose the SINR of a user $u$ in this case is represented by $\gamma_{u, M}$;

$$
\gamma_{u, M}=\frac{P_{S(u)} L_{S(u)}\left(\vec{q}_{u}\right)}{N+\sum_{c \neq S(u)} \rho_{c} P_{c} L_{c}\left(\vec{q}_{u}\right)} .
$$

By applying range extension, more users are connected to the picocell and the cell load in the picocell is expected to increase. To facilitate the analysis, the interference from picocell is separated from the total interference in (16). Denoting the picocell as $p$, the SINR of user $u$ can be rewritten as

$$
\begin{aligned}
\gamma_{u, M} & =\frac{P_{S(u)} L_{S(u)}\left(\vec{q}_{u}\right)}{N+\sum_{c \neq S(u)} \rho_{c} P_{c} L_{c}\left(\vec{q}_{u}\right)} \\
& =\frac{P_{S(u)} L_{S(u)}\left(\vec{q}_{u}\right)}{N+\sum_{\substack{c \neq S(u) \\
c \neq p}} \rho_{c} P_{c} L_{c}\left(\vec{q}_{u}\right)+\rho_{p} P_{p} L_{p}\left(\vec{q}_{u}\right)} .
\end{aligned}
$$

Denote the SINR of user $u$ after CRE as $\gamma_{u, M}^{\prime}$. After applying CRE, the received signal power from the serving macrocell can be assumed to be unchanged if we assume the user's position does not change significantly during the time of handover execution. The interference from other cells except for the picocell can also be assumed to be unchanged for simplicity. However, with CRE, the load in the picocell, $\rho_{p}^{\prime}$, is expected to increase $\left(\rho_{p}^{\prime}>\rho_{p}\right)$. Consequently, the interference from picocell will increase eventually. The SINR of user $u$ after CRE is then

$$
\gamma_{u, M}^{\prime}=\frac{P_{S(u)} L_{S(u)}\left(\vec{q}_{u}\right)}{N+\sum_{\substack{c \neq S(u) \\ c \neq p}} \rho_{c} P_{c} L_{c}\left(\vec{q}_{u}\right)+\rho_{p}^{\prime} P_{p} L_{p}\left(\vec{q}_{u}\right)} .
$$

Therefore, the SINR of users served by the macrocell will decrease due to the increased interference from picocell,

$$
\gamma_{u, M}^{\prime}<\gamma_{u, M}
$$

Denoting the macrocell as $m$ and the capacity of users in macrocell as $C_{M}(u)$, the capacity is determined by both SINR of these users and the load situation in the macrocell:

$$
C_{M}(u)=\left(1-\rho_{m}\right) \log _{2}\left(1+\gamma_{u, M}\right) .
$$
have

Denoting the capacity of user $u$ after CRE as $C_{M}^{\prime}(u)$, we

$$
C_{M}^{\prime}(u)=\left(1-\rho_{m}^{\prime}\right) \log _{2}\left(1+\gamma_{u, M}^{\prime}\right)
$$

It is desirable that, with CRE, the capacity of users stay in macrocell could increase:

$$
\begin{gathered}
C_{M}^{\prime}(u)>C_{M}(u) \\
\left(1-\rho_{m}^{\prime}\right) \log _{2}\left(1+\gamma_{u, M}^{\prime}\right)>\left(1-\rho_{m}\right) \log _{2}\left(1+\gamma_{u, M}\right)
\end{gathered}
$$

Therefore, we can derive the theorem as below to show the condition that ensures a gain in capacity with CRE for users stay in macrocell.

Theorem 2. The capacity of users in macrocell can be increased if and only if the cell load in the macrocell after CRE satisfies the inequality as follows:

$$
\rho_{m}^{\prime}<1-\left(1-\rho_{m}\right) \frac{\log _{2}\left(1+\gamma_{u, M}\right)}{\log _{2}\left(1+\gamma_{u, M}^{\prime}\right)}
$$

Theorem 2 shows that only when the cell load of macrocell decreases low enough to compensate for the loss in SINR, the capacity of these users can increase.

More specifically, suppose the number of users in the macrocell is $N_{M}$, the number of scheduled resource blocks for each user $u$ is $\mathrm{NRB}_{u}$, and the total number of available resource blocks in the cell is $\mathrm{NRB}_{\text {avail, } m}$; then the macrocell load $\rho_{m}$ is

$$
\rho_{m}=\frac{\sum_{u=1}^{N_{M}} \mathrm{NRB}_{u}}{\mathrm{NRB}_{\mathrm{avail}, m}}
$$

Therefore, the capacity of user $u$ is

$$
C_{M}(u)=\left(1-\frac{\sum_{u=1}^{N_{M}} \mathrm{NRB}_{u}}{\mathrm{NRB}_{\text {avail }, m}}\right) \log _{2}\left(1+\gamma_{u, M}\right) .
$$

The number of scheduled resource blocks is usually a function of SINR. Considering a typical traffic type that is named as constant bit rate (CBR) service as the example,

$$
\mathrm{NRB}_{u}=\frac{D_{u}}{R\left(\gamma_{u, M}\right) \cdot \mathrm{BW}}
$$

where BW is the bandwidth of one PRB (e.g., in LTE system, BW is $180 \mathrm{kHz}$ ) and $D_{u}$ is the demanded data rate of user $u$. As shown in (4), $R(\gamma)$ is the achievable data rate that can be estimated according to the Shannon capacity. Therefore, the capacity of users stay in macrocell is actually a function of both SINR and the number of users in macrocell. Considering that the served user number linearly contributes to the capacity while SINR contributes in logarithmic scale, from the capacity of macrocell point of view, it is desirable to handover more users out of macrocell. However, from the overall capacity point of view, it is necessary to consider the capacity change of both users stay in macrocell and users stay in CRE region as well as users in picocell which we will analyze in the following section. 

have

Suppose the total capacity of users in macrocell is $C_{M}$; we

$$
\begin{aligned}
C_{M} & =\sum_{u=1}^{N_{M}} C_{M}(u) \\
& =\sum_{u=1}^{N_{M}}\left(1-\frac{\sum_{u=1}^{N_{M}} \mathrm{NRB}_{u}}{\mathrm{NRB}_{\text {avail }, m}}\right) \log _{2}\left(1+\gamma_{u, M}\right) .
\end{aligned}
$$

2.2.3. Capacity Analysis for Users in Picocell. The capacity of users served by picocell both before and after applying CRE is analyzed similarly. For these users, although the serving cell is unchanged, the SINR and capacity are eventually impacted by range extension.

Suppose the SINR of a user $u$ in this case is presented by $\gamma_{u, P}$;

$$
\gamma_{u, P}=\frac{P_{S(u)} L_{S(u)}\left(\vec{q}_{u}\right)}{N+\sum_{c \neq S(u)} \rho_{c} P_{c} L_{c}\left(\overrightarrow{q_{u}}\right)} .
$$

To facilitate the analysis, the macrocell interference is separated from the total interference in (28). Denoting the macrocell as $m$, the SINR of user $u$ can be rewritten as

$$
\begin{aligned}
\gamma_{u, P} & =\frac{P_{S(u)} L_{S(u)}\left(\vec{q}_{u}\right)}{N+\sum_{c \neq S(u)} \rho_{c} P_{c} L_{c}\left(\vec{q}_{u}\right)} \\
& =\frac{P_{S(u)} L_{S(u)}\left(\vec{q}_{u}\right)}{N+\sum_{\substack{c \neq S(u) \\
c \neq m}} \rho_{c} P_{c} L_{c}\left(\vec{q}_{u}\right)+\rho_{m} P_{m} L_{m}\left(\vec{q}_{u}\right)} .
\end{aligned}
$$

Denote the SINR of user $u$ after CRE as $\gamma_{u, P}^{\prime}$. Similar to the analysis for users in macrocell, after applying CRE, the received signal power from the serving picocell can be assumed to be unchanged and the interference from other cells except for the macrocell can also be assumed to be unchanged for simplicity. However, with CRE, the load in the macrocell, denoted by $\rho_{m}^{\prime}$, is changed and usually the load in macrocell is expected to be reduced $\left(\rho_{m}^{\prime}<\rho_{m}\right)$. Consequently, the interference from macrocell will decrease. The SINR of pico user $u$ after CRE is then

$$
\gamma_{u, P}^{\prime}=\frac{P_{S(u)} L_{S(u)}\left(\vec{q}_{u}\right)}{N+\sum_{\substack{c \neq S(u) \\ c \neq m}} \rho_{c} P_{c} L_{c}\left(\vec{q}_{u}\right)+\rho_{m}^{\prime} P_{m} L_{m}\left(\vec{q}_{u}\right)}
$$

Therefore, the SINR of users served by picocell increases due to the reduced interference, as

$$
\gamma_{u, P}^{\prime}>\gamma_{u, P}
$$

Denoting the picocell as $p$ and the capacity of user $u$ in picocell with and without $\mathrm{CRE}$ as $C_{p}(u)$ and $C_{p}^{\prime}(u)$, respectively, then

$$
\begin{aligned}
& C_{P}(u)=\left(1-\rho_{P}\right) \log _{2}\left(1+\gamma_{u, P}\right), \\
& C_{P}^{\prime}(u)=\left(1-\rho_{P}^{\prime}\right) \log _{2}\left(1+\gamma_{u, P}^{\prime}\right) .
\end{aligned}
$$

To achieve capacity gain for users in picocell, that is,

$$
\begin{gathered}
C_{P}^{\prime}(u)>C_{P}(u), \\
\left(1-\rho_{P}^{\prime}\right) \log _{2}\left(1+\gamma_{u, P}^{\prime}\right)>\left(1-\rho_{P}\right) \log _{2}\left(1+\gamma_{u, P}\right) .
\end{gathered}
$$

Therefore, we derive the following theorem which states the conditions for users in picocell to achieve capacity gain by CRE.

Theorem 3. The capacity of users in picocell can be increased if and only if the cell load in the picocell after CRE satisfies the inequality as follows:

$$
\rho_{p}^{\prime}<1-\left(1-\rho_{P}\right) \frac{\log _{2}\left(1+\gamma_{u, P}\right)}{\log _{2}\left(1+\gamma_{u, P}^{\prime}\right)} .
$$

From Theorem 3, it can be concluded that if the increased cell load in picocell does not exceed the limit as shown in inequality (35), the capacity of users in picocell can increase. Otherwise, the capacity of the pico users would decrease.

Suppose the number of users in the picocell is $N_{P}$, the number of scheduled resource blocks for each user $u$ is $\mathrm{NRB}_{u}$, and the total number of available resource blocks in the picocell is $\mathrm{NRB}_{\text {avail, } p}$. The picocell load $\rho_{p}$ is then

$$
\rho_{p}=\frac{\sum_{u=1}^{N_{p}} \mathrm{NRB}_{u}}{\mathrm{NRB}_{\text {avail }, p}}
$$

And the picocell load after CRE is

$$
\rho_{p}^{\prime}=\frac{\sum_{u=1}^{N_{P}+N_{R}} \mathrm{NRB}_{u}^{\prime}}{\mathrm{NRB}_{\mathrm{avail}, p}}
$$

where $N_{R}$ is the number of users in CRE region and $\mathrm{NRB}_{u}^{\prime}$ is the number of resource blocks of user $u$ served by pico. As shown in (26), the number of resource blocks of user $u$ usually depends on its SINR. According to the analysis, typically, the SINR of users in CRE region would decrease while the SINR of users in picocell would increase, which results in either increased NRB or decreased NRB. However, considering the increased number of served users, $N_{R}$, the cell load in picocell usually gets difficult to satisfy the inequality of (35) and results in a decreased capacity.

Suppose the total capacity of users in picocell is $C_{P}$; we have

$$
\begin{aligned}
C_{P} & =\sum_{u=1}^{N_{P}} C_{P}(u) \\
& =\sum_{u=1}^{N_{P}}\left(1-\frac{\sum_{u=1}^{N_{P}} \mathrm{NRB}_{u}}{\mathrm{NRB}_{\text {avail }, P}}\right) \log _{2}\left(1+\gamma_{u, P}\right) .
\end{aligned}
$$

2.2.4. Overall Capacity. Summarizing capacity of users in macrocell, in picocell, and in the CRE region, we can get the overall capacity as

$$
C_{\text {total }}=C_{M}+C_{P}+C_{\mathrm{CRE}}
$$


From the analysis above, it can be observed that with CRE the overall capacity not only depends on the change of SINRs of users in macrocell, in picocell, and in CRE region but also depends on the load situation and change in both macrocell and picocell. CRE may not always give benefit to the overall capacity in the system. Considering an example that if the traffic load in macrocell is low and there are enough radio resources that can be allocated to the served users, the throughput of these users is consequently limited by their channel conditions, that is, SINRs. In this case, forcing these users into the picocell by CRE can further damage the SINRs and consequently damage the users' throughput. On the other hand, if the traffic load in macrocell is high and the users' throughputs are limited by the available radio resource, handing over these users to LPN by CRE can improve the users' throughputs.

To get the optimal overall capacity, in the next section, an adaptive algorithm to optimize the CSO setting via prediction of overall capacity for different CSO values is proposed.

\section{Capacity Optimization Algorithm with Adaptive Cell Range Control}

In this section, an adaptive algorithm is proposed to optimize the overall capacity by adjusting the CSO setting. In this algorithm, the CSO setting is updated periodically. At each update instance, the current CSO value is updated by a new value if the predicted overall capacity can be increased by this new attempting value. The attempting CSO setting is obtained by increasing or decreasing a step to the current CSO. The new CSO value which gives the best overall capacity is then selected. The algorithm is described step by step as in below.

3.1. Step 1: User Grouping with Attempting CSO Settings. Suppose CSO updating step is $\Delta_{\mathrm{CSO}}$. At each update instance $t$, CSO value is updated based on current $\mathrm{CSO}$ value, $\mathrm{CSO}_{t-1}$, which was set at time interval $t-1$. The attempting CSO value, $\mathrm{CSO}_{t \text {, att }}$, is obtained by either increasing or decreasing $\mathrm{CSO}_{t-1}$ by the updating step $\Delta_{\mathrm{CSO}}$, as

$$
\begin{aligned}
& \mathrm{CSO}_{t, \text { att }}=\mathrm{CSO}_{t, \text { inc }}=\mathrm{CSO}_{t-1}+\Delta_{\mathrm{CSO}} \\
& \mathrm{CSO}_{t, \text { att }}=\mathrm{CSO}_{t, \text { dec }}=\mathrm{CSO}_{t-1}-\Delta_{\mathrm{CSO}} .
\end{aligned}
$$

Based on the RSRP measurements from both macrocell and picocell, for the attempted CSO setting, all of the users can be divided into three groups.

Group 1. Macro users. These users stay in the macrocell both with the previous CSO setting and the new CSO setting:

$$
\begin{aligned}
& \operatorname{RSRP}_{u}^{p}+\operatorname{CSO}_{t-1} \leq \operatorname{RSRP}_{u}^{m}, \\
& \operatorname{RSRP}_{u}^{p}+\operatorname{CSO}_{t, \text { att }} \leq \operatorname{RSRP}_{u}^{m} .
\end{aligned}
$$

Group 2. Pico users. These users stay in the picocell both with the previous CSO setting and the new CSO setting:

$$
\begin{aligned}
& \operatorname{RSRP}_{u}^{p}+\mathrm{CSO}_{t-1}>\operatorname{RSRP}_{u}^{m} \\
& \operatorname{RSRP}_{u}^{p}+\mathrm{CSO}_{t, \text { att }}>\operatorname{RSRP}_{u}^{m} .
\end{aligned}
$$

Group 3. Users in CRE region. These users will change their serving cell with new CSO setting. Take the case that CSO is increased as the following example:

$$
\begin{aligned}
& \operatorname{RSRP}_{u}^{p}+\operatorname{CSO}_{t-1} \leq \operatorname{RSRP}_{u}^{m}, \\
& \operatorname{RSRP}_{u}^{p}+\operatorname{CSO}_{t, \text { att }}>\operatorname{RSRP}_{u}^{m} .
\end{aligned}
$$

On the basis of the user grouping, the capacities of users in different groups are predicted, respectively, and the overall capacity is predicted consequently.

3.2. Step 2: Capacity Prediction with Attempting CSO Settings. In step 2, the capacities of users in different groups are predicted, respectively, according to the predicted SINR and cell load. More details can be found below.

3.2.1. Step 2.1: Capacity Prediction for Users in CRE Region. To estimate the new capacity of users in CRE region, we need to estimate the new SINR and predict the new cell load in picocell and in macrocell.

Taking the case that the CSO is increased by a step as example, to estimate the new SINR in picocell, $\gamma_{u, \text { CRE }}^{(p)}$, we can use

$$
\gamma_{u, \mathrm{CRE}}^{(p)}=\frac{\operatorname{RSRP}^{(p)}}{\left(\operatorname{RSRP}^{(m)} / \gamma_{u, \mathrm{CRE}}^{(m)}\right)+\operatorname{RSRP}^{(m)}-\operatorname{RSRP}^{(p)}}
$$

To estimate the new picocell load, we need to estimate the required number of RBs according to the new SINR. For CBR service, this can be estimated similarly to (26) by

$$
N_{u}=\frac{D_{u}}{R\left(\gamma_{u, \mathrm{CRE}}^{(p)}\right) \cdot \mathrm{BW}} .
$$

For services other than CBR service, the new required number of RBs can be estimated according to the relative relationship between the previous SINR in macrocell, previous required number of RBs in macrocell, and the predicted SINR in picocell:

$$
N_{u}^{\text {new }}=N_{u}^{\text {old }} \cdot \frac{R\left(\gamma_{u, \mathrm{CRE}}^{(m)}\right)}{R\left(\gamma_{u, \mathrm{CRE}}^{(p)}\right)}
$$

Based on the prediction of the required number of RBs, the new picocell load can be estimated by assuming the required number of $\mathrm{RBs}$ is unchanged for other users in picocell:

$$
\rho_{p}^{\text {new }}=\frac{\sum_{u=1}^{N_{P}} N_{u}+\sum_{u=1}^{N_{R}} N_{u}{ }^{\text {new }}}{\mathrm{NRB}_{\text {avail }, p}} .
$$


Consequently, the new capacity of users newly added to CRE region can be estimated according to the analysis in Section 2 .

\subsubsection{Step 2.2: Capacity Prediction for Macro Users and Pico} Users. For macro users, to predict the SINR of users with new CSO, the picocell load with new CSO needs to be estimated. This can be achieved by method described in Step 2.1.

The new cell load in macrocell can be simply estimated by the new number of served users and assuming that the required numbers of RBs for macro users are unchanged.

The capacity of users in picocell can be estimated similarly.

3.3. Optimal CSO Selection and Updating. The predicted new capacities of users in different groups are summed together to get the overall predicted capacity. The predicted overall capacity is then compared with current capacity. If the estimated new capacity is larger than the current capacity, the CSO is updated:

$$
\text { If } \mathrm{C}\left(\mathrm{CSO}_{t, \text { att }}\right)>C\left(\mathrm{CSO}_{t-1}\right), \mathrm{CSO}_{t}=\mathrm{CSO}_{t, \text { att }} \text {. }
$$

Otherwise, CSO keeps unchanged.

If the CSO is decreased by a step, the overall capacity can be estimated similarly by reversing the calculation shown above. If the new CSO value gives better predicted performance, the CSO value is updated.

With the proposed adaptive algorithm, the CSO value is adjusted according to the predicted overall capacity in both macrocell and picocell. By selecting the most suitable CSO value, the overall capacity is expected to be optimized.

\section{Numerical Results}

The proposed adaptive CSO updating algorithm is evaluated via system-level simulations. In this section the simulation results are presented and discussed. The simulation assumptions and parameter settings are illustrated in Table 1.

Two typical scenarios are tested in the evaluation. In scenario 1 , the users are uniformly distributed in the entire simulated area. LPNs are deployed randomly. In scenario 2, LPNs are deployed in hotspot areas, and the hotspot areas take nearly $60 \%$ of the users. The base station deployment and user distribution in two scenarios are illustrated in Figures 3 and 4 . To have a clear view, only part of the simulated areas is illustrated.

The percentages of users belonging to macrocell or LPNs in the two scenarios are illustrated in Figure 5 with different CSO settings. It can be observed that, when CSO setting increases, the users belonging to LPNs increase. It can be observed as well that the percentages of users belonging to LPNs in hotspot scenario are much higher than in uniform scenario.

Figure 6 shows the change of average cell load with different CSO settings in different scenarios. In uniform scenario, most of the users are in macrocells, and the load in the macrocell is consequently quite high; the cell load in picocell increases with the increase in the CSO setting. In

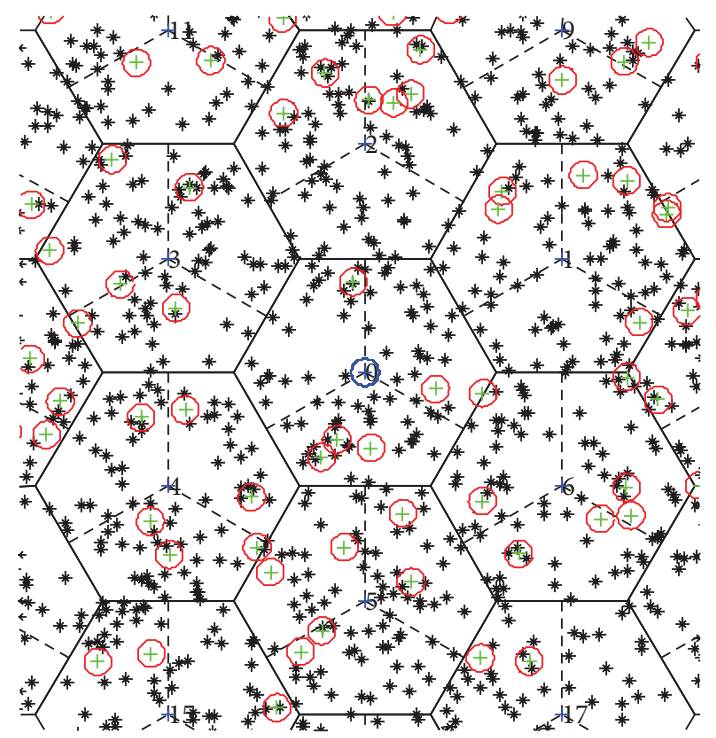

FIgURE 3: Cell layout and user distribution in uniform scenario.

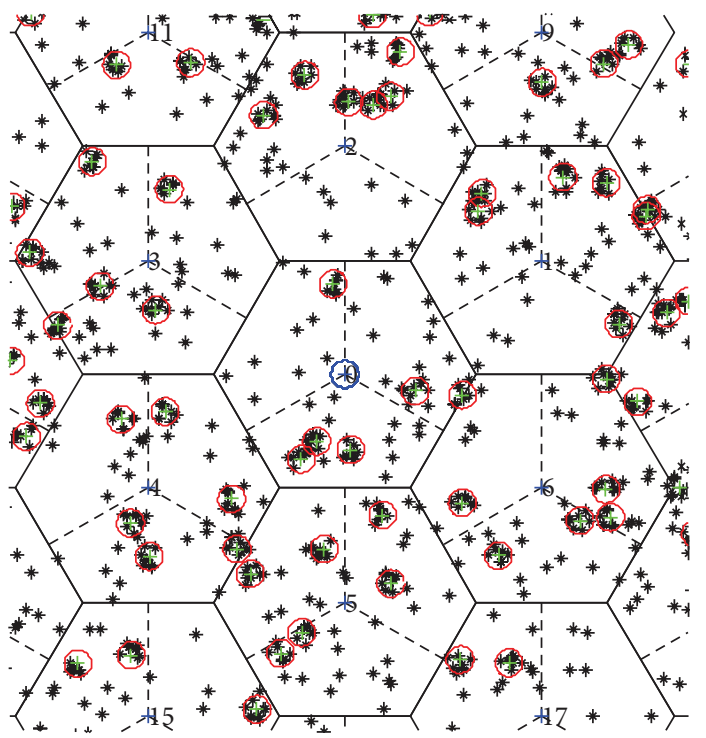

FIGURE 4: Cell layout and user distribution in hotspot scenario.

hotspot scenario, the average cell load in macrocells decreases with the increase in CSO setting while the average cell load in LPNs increases further with the increased CSO setting.

In Figure 7, the average sector throughput gain with different CSO settings is compared with the proposed adaptive algorithm for uniform scenario. The average sector throughput with $\mathrm{CSO}=0 \mathrm{~dB}$ is used as reference to obtain the average sector throughput gain. It can be observed that, in this scenario, a higher CSO setting is desirable to offload more users to LPNs. The proposed adaptive algorithm can achieve the similar throughput to the throughput of the optimal CSO setting.

In Figure 8, the average sector throughput gain with different CSO settings is compared with the proposed adaptive algorithm in hotspot scenario. The average sector throughput 


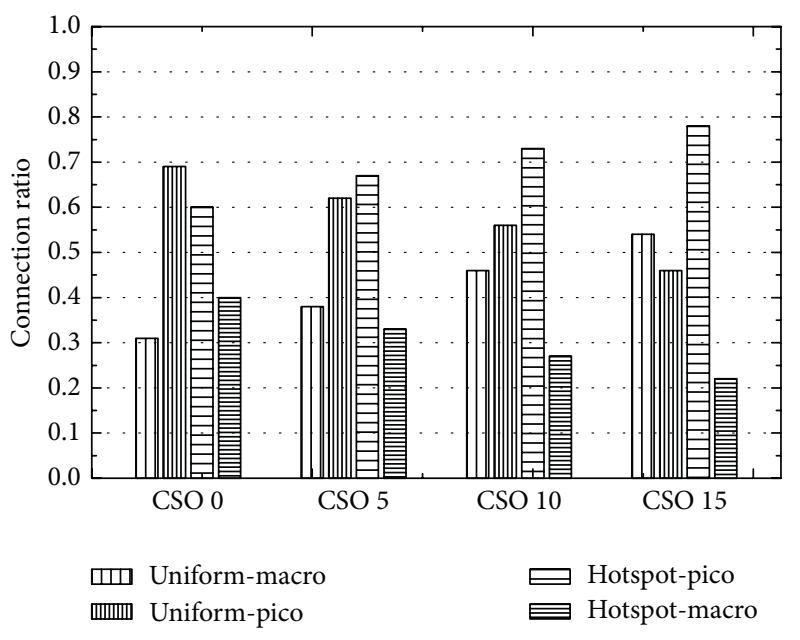

FIgURE 5: Connection ratio with different CSOs.

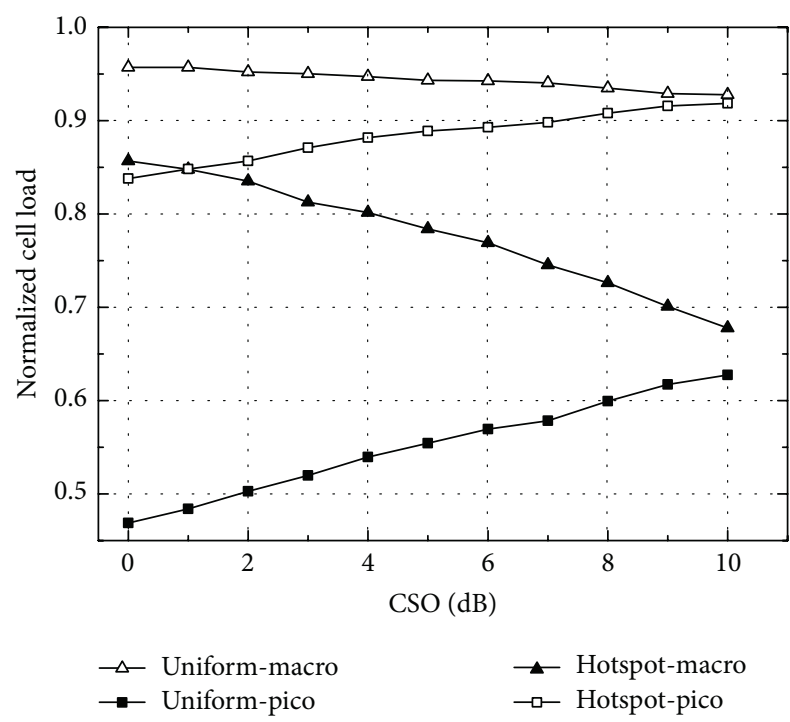

FIgURE 6: Cell load with different CSOs.

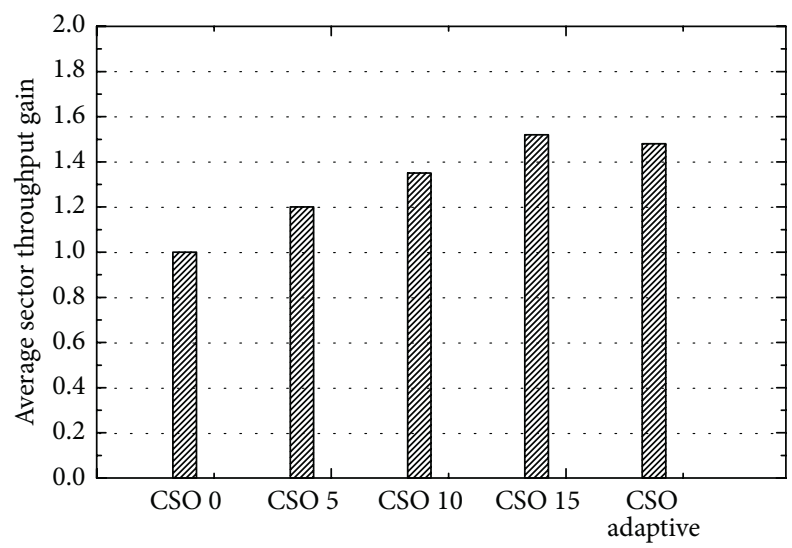

FIGURE 7: Average sector throughput gain in uniform scenario.

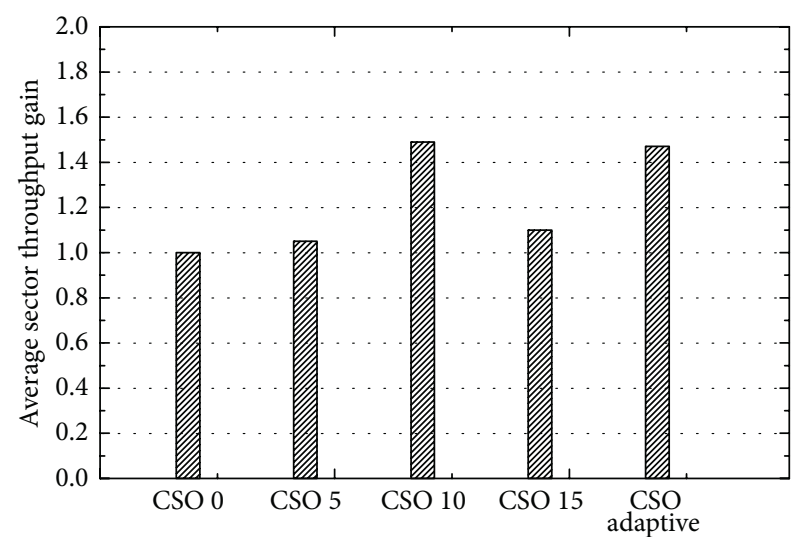

Figure 8: Average sector throughput gain in hotspot scenario.

with $\mathrm{CSO}=0 \mathrm{~dB}$ is used as reference to obtain the average sector throughput gain. In this scenario, CSO of $10 \mathrm{~dB}$ is desirable to balance the offloading gain and SINR loss. The proposed algorithm achieves the similar performance to the optimal CSO setting.

In different scenarios, the optimal CSO setting is different. However, the proposed adaptive algorithm can achieve similar performance with the optimal CSO setting in both scenarios.

\section{Conclusion}

As an attractive means of expanding mobile network capacity, heterogeneous network has been included in LTE-advanced. Cell range extension is an important feature in heterogeneous network to improve the utilization of the resource of low power nodes. However, the users in the cell range extension area usually suffer from high interference from macrocells. To avoid the unnecessary interference, it is desirable to carefully consider the optimal cell selection offset value. In this paper, the capacity of users in macrocell and small cells is analyzed thoroughly. The conditions to improve capacity via cell range extension are derived, respectively, for users both in macrocell and in small cell. Based on the analysis, an adaptive small cell coverage control algorithm is proposed. With the proposed adaptive algorithm, the cell selection offset is updated periodically based on the prediction of the overall system capacity. The algorithm is evaluated by systemlevel simulations, and the results indicate that, with the proposed algorithm, a nearly optimal system performance can be achieved in all tested load cases.

\section{Conflict of Interests}

The authors declare that there is no conflict of interests regarding the publication of this paper. 


\section{Acknowledgments}

This work was supported by the State Major Science and Technology Special Projects (Grant no. 2013ZX03001026001) and the Fundamental Research Funds for the Central Universities (Grant no. 2014RC0107).

\section{References}

[1] A. Damnjanovic, J. Montojo, Y. Wei et al., "A survey on 3GPP heterogeneous networks," IEEE Wireless Communications, vol. 18, no. 3, pp. 10-21, 2011.

[2] E. Dahlman, S. Parkvall, and J. Skold, 4G LTE/LTE-Advanced for Mobile Broadband, Academic Press, 2011.

[3] A. Ghosh, R. Ratasuk, B. Mondal, N. Mangalvedhe, and T. Thomas, "LTE-advanced: next-generation wireless broadband technology," IEEE Wireless Communications, vol. 17, no. 3, pp. 10-22, 2010.

[4] X. Zhang, X. Gu, W. Li, L. Zhang, J. Shen, and Y. Wan, “The study of indoor and field trials on $2 \times 8$ MIMO architecture in TD-LTE network," International Journal of Antennas and Propagations, vol. 2013, Article ID 181579, 9 pages, 2013.

[5] G. Yuan, X. Zhang, W. Wang, and Y. Yang, "Carrier aggregation for LTE-advanced mobile communication systems," IEEE Communications Magazine, vol. 48, no. 2, pp. 88-93, 2010.

[6] R. Irmer, H. Droste, P. Marsch et al., "Coordinated multipoint: concepts, performance, and field trial results," IEEE Communications Magazine, vol. 49, no. 2, pp. 102-111, 2011.

[7] I. Siomina and Y. Di, "Load balancing in heterogeneous LTE: range optimization via cell offset and load-coupling characterization," in Proceedings of the IEEE International Conference on Communications, pp. 1357-1361, 2012.

[8] The 3rd Generation Partnership Project (3GPP), "Feasibility study for further advancements for E-UTRA (LTEAdvanced), (Release 10)," Tech. Rep. TR 36.912, 2011.

[9] The 3rd Generation Partnership Project(3GPP), "System performance of heterogeneous networks with range expansion," Tech. Rep. R1-101203, Samsung, 2010.

[10] I. Guvenc, "Capacity and fairness analysis of heterogeneous networks with range expansion and interference coordination," IEEE Communications Letters, vol. 15, no. 10, pp. 1084-1087, 2011.

[11] H-S. Jo, Y. J. Sang, P. Xia, and J. G. Andrews, "Heterogeneous cellular networks with flexible cell association: a comprehensive downlink SINR analysis," IEEE Transactions on Wireless Communications, vol. 11, no. 10, pp. 3484-3495, 2012.

[12] "Self-optimisationand self-configuration in wireless networks," SOCRATES, European Research Project, http://www.fp7socrates.org.

[13] The 3rd Generation Partnership Project(3GPP), "Self-configuring and self-optimizing network use cases and solutions," Tech. Rep. TR 36.902, 2009.

[14] "Use cases related to Self Organizing network, Overall description," Next generation Mobile Networks, http://www.ngmn.org.

[15] X. Chu and D. Lopez-Perez, Heterogeneous Cellular Networks Theory, Simulation and Deployment, Cambridge University Press, 2013.

[16] P. Tian, H. Tian, J. Zhu, L. Chen, and X. She, "An adaptive bias configuration strategy for range extension in LTE-Advanced heterogeneous networks," in Proceedings of the IET International Conference on Communication Technology and Application (ICCTA '11), pp. 336-340, 2011.
[17] K. Kikuchi and H. Otsuka, "Proposal of adaptive control CRE in heterogeneous networks," in Proceedings of the IEEE International Symposium on Personal Indoor and Mobile Radio Communications (PIMRC '12), pp. 910-914, 2012.

[18] I. Viering, M. Döttling, and A. Lobinger, "A mathematical perspective of self-optimizing wireless networks," in Proceedings of the IEEE International Conference on Communications (ICC '09), pp. 1-6, June 2009.

[19] A. Lobinger, S. Stefanski, T. Jansen, and I. Balan, "Load balancing in downlink LTE self-optimizing networks," in Proceedings of the IEEE 71st Vehicular Technology Conference (VTC '10), pp. 1-5, May 2010.

[20] "Guidelines for evaluation of radio transmission technologies for IMT-2000," Recommendation ITU-R M.1225, 1997.

[21] The 3rd Generation Partnership Project (3GPP), "Further advancements for E-UTRAN physical layer aspects, (Release 9)," Tech. Rep. TS 36.814, 2010. 

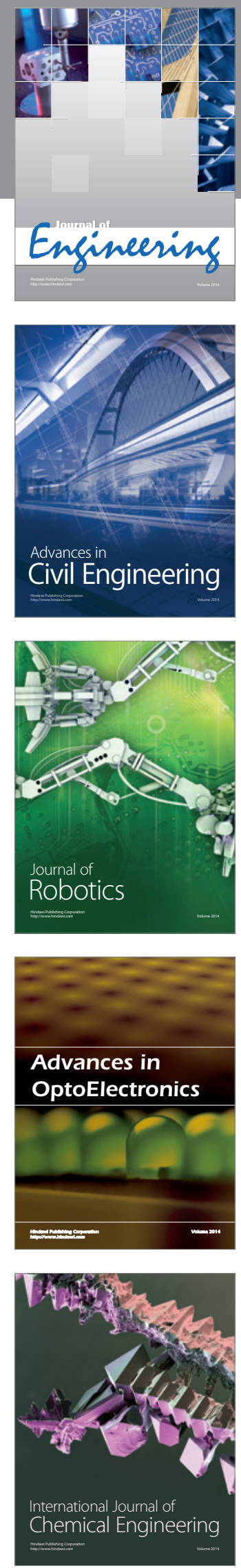

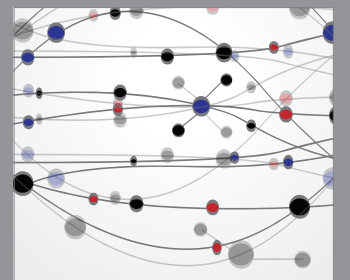

The Scientific World Journal
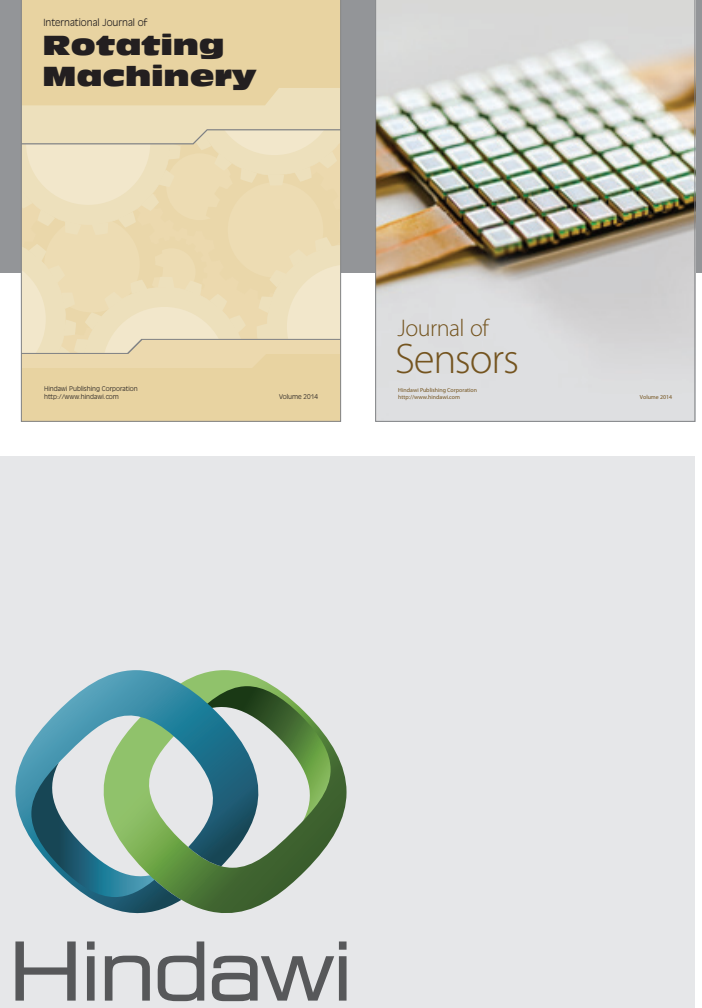

Submit your manuscripts at http://www.hindawi.com
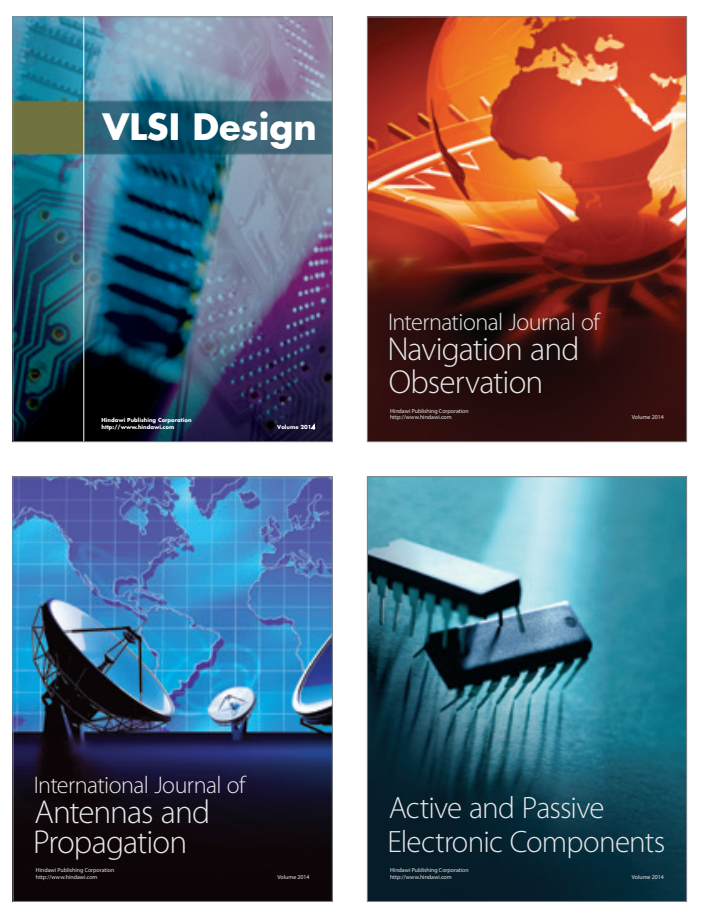
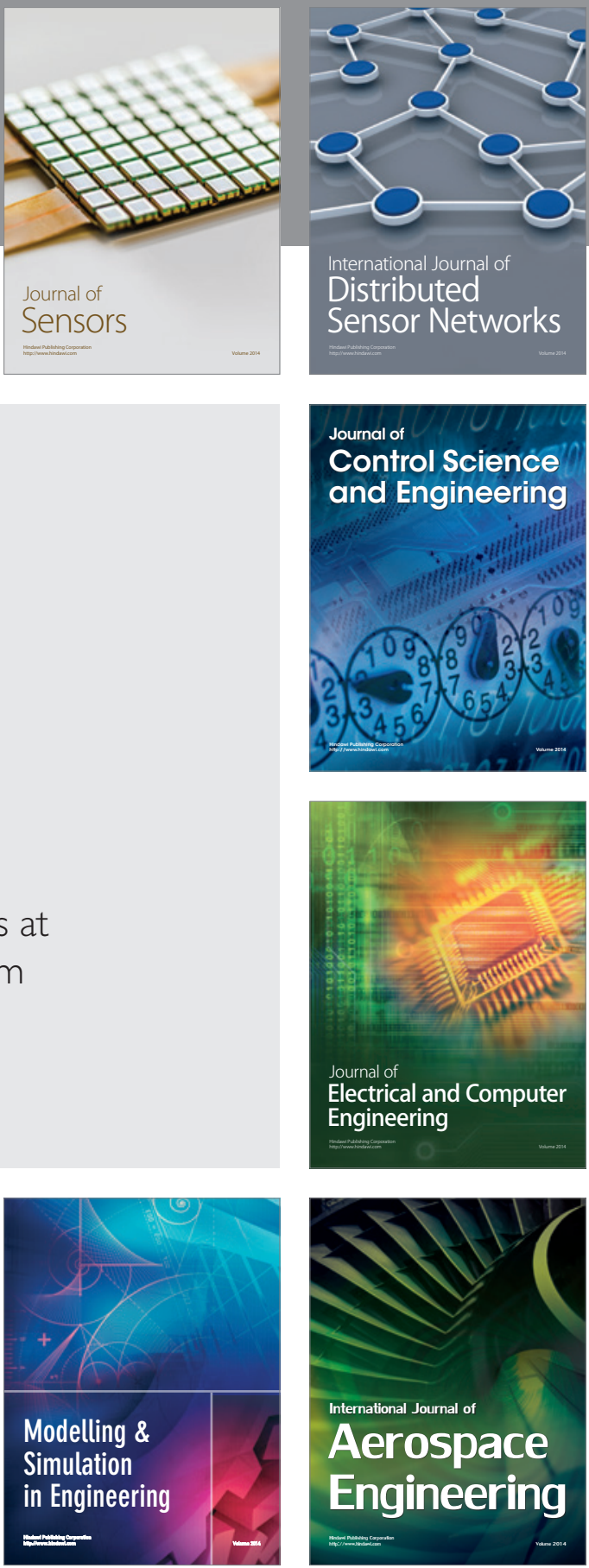

Journal of

Control Science

and Engineering
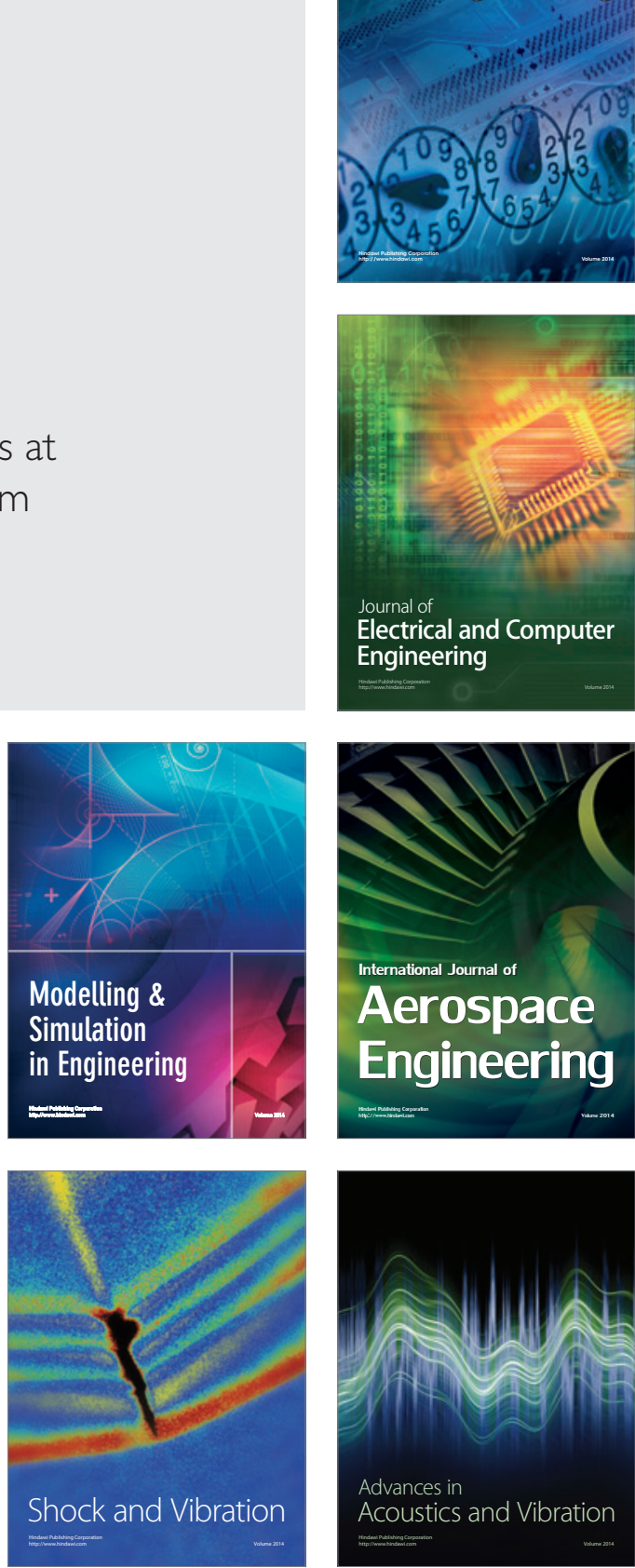